The University of Maine

\title{
DigitalCommons@UMaine
}

Psychology Faculty Scholarship

Psychology

$1-1990$

\section{Emotional Processing in the Treatment of Simple Phobia: A Comparison of Imaginal and In Vivo Exposure}

Jeffrey E. Hecker

University of Maine - Main, jeff.hecker@umit.maine.edu

Follow this and additional works at: https://digitalcommons.library.umaine.edu/psy_facpub

Part of the Psychology Commons

\section{Repository Citation}

Hecker, Jeffrey E., "Emotional Processing in the Treatment of Simple Phobia: A Comparison of Imaginal and In Vivo Exposure" (1990). Psychology Faculty Scholarship. 17.

https://digitalcommons.library.umaine.edu/psy_facpub/17

This Article is brought to you for free and open access by DigitalCommons@UMaine. It has been accepted for inclusion in Psychology Faculty Scholarship by an authorized administrator of DigitalCommons@UMaine. For more information, please contact um.library.technical.services@maine.edu. 


\title{
Emotional Processing in the Treatment of Simple Phobia: A Comparison of Imaginal and In Vivo Exposure
}

\author{
Jeffrey E. Hecker
}

\author{
University of Maine, \\ Department of Psychology \\ 301 Little Hall \\ Orono, ME 04469
}

Two groups of moderately snake phobic college students were given either imaginal or in vivo exposure treatment. The groups were compared on self-report and physiological measures of fear activation during exposure trials, as well as on within- and across-session habituation of fear responses. On these measures, as well as on treatment outcome, the two groups were found to be very similar. The results lend further support to the importance of the concept of emotional processing in understanding fear reduction processes. Differences in treatment procedure may be important only when one procedure facilitates emotional processing more than another.

\section{Introduction}

In a earlier article in this journal, we argued that bioinformational theory (Lang, 1979, 1984, 1985) and the concept of emotional processing (Foa \& Kozak, 1986) provided a useful framework from which to evaluate the literature comparing imaginal and in vivo flooding (Hecker and Thorpe, 1987).

In bioinformational theory, Lang argues that a phobia is represented in long-term memory as a network of propositionally coded information. The propositions can be organized into three types: Stimulus propositions which describe the fear-evoking stimuli; response propositions, which contain information about physiological, overt behavioural and verbal-cognitive responses; and meaning propositions, which contain interpretive information about the stimulus and response elements. This network is thought to be a prototype for overt emotional expression. Fear reduction involves dismantling this phobia network or fear structure. Exposure to phobic stimuli and imagining phobic situations facilitate processing of the network and it is during processing that the structure is broken down (see Lang, 1984). 
Emotional processing refers to changes that occur to the fear structure during exposure therapy. Foa and Kozak identified three indices of emotional processing: activation of fear responses, their habituation within treatment sessions, and their habituation across treatment sessions. Activation can be inferred from physiological arousal that is consistent with the fear response (e.g., increased heart rate) and/or self-reported fear during exposure. Habituation of fear responses is indicated by a decrease in fear within exposure sessions. Across-session habituation is indicated by a decrease in the activation of fear in response to the initial presentation of phobic stimuli during subsequent treatment sessions. By examining the available studies comparing imaginal and in vivo flooding with respect to the indices of emotional processing, we were able to show that fear reduction was related to the degree of processing that occurred during treatment (Hecker and Thorpe, 1987). Procedural differences (i.e., imaginal versus in vivo exposure) were not as important as whether or not the procedure engendered emotional processing.

The present study was designed to examine emotional processing during imaginal and in vivo exposure treatments of a simple phobia. Activation of fear responses and their habituation within and across sessions were recorded during each procedure and the treatments were compared on measures of emotional processing.

\section{Method}

The data for this study were obtained in the context of a larger experiment which compared various forms of imaginal and in vivo exposure in the treatment of simple phobics (Hecker, Port and Thorpe, 1986).

\section{Subjects}

Subjects were 43 female undergraduates who reported a significant fear of snakes and who volunteered to participate in this study from a choice of several as part of an introductory psychology course. Five subjects did not meet minimal selection criteria for snake-fear (i.e., they completed all steps of the pre-treatment behavioural test). The data for two subjects in the imaginal exposure condition were lost due to experimenter error. Thirty-six subjects, therefore, produced useful data. Seventeen subjects were in the imaginal exposure condition. Nineteen were in the in vivo exposure condition.

\section{Apparatus}

An Exersentry (Model EX-3), available from Respironics, Inc. U.S.A., was used to measure heart rate. The device is a portable monitoring system that provides a digital display of average heart rate in beats per minute based on a four beat estimate. Heart rate was recorded by an experimenter in an adjoining room. 


\section{Procedure}

Subjects were seen individually for pre-treatment assessment. Fear of snakes was assessed in five ways. Two paper-and-pencil, self-report measures were used: The Snake Phobia Questionnaire (SPQ) is a 30-item, true-false, measure of snake phobia (Klorman et al., 1974); The Snake Fearfulness Rating (SNFR) is a five point global rating of snake fear ( $1=$ very little; $5=$ very much). Three measures were also gathered during a behavioural test with a live snake: Subjects' scores on the Behavioural Test-Avoidance measure (BTAV) were determined by the number of steps completed $(0=$ maximum avoidance; $8=$ no avoidance); Subjects also rated their level of fear during each completed step of the behavioural test on a nine point scale. The rating of their level of fear during the farthest step completed determined their score on the Behavioural Test-Fear Rating measure (BTFR). Subjects' heart rate was also recorded during each step of the behavioural test completed. The difference between their heart rate during the last completed step and their heart rate during a baseline assessment determined their score on the Behavioural Test-Heart Rate measure (BTHR).

The behavioural test proceeded as follows. Subjects put on the Exersentry themselves. They were seated in a comfortable chair and asked to relax while a baseline measure of their heart rate was recorded. The snake was housed in a 19 liter aquarium with glass on four sides and the bottom and a Plexiglas top. Prior to each step of the behavioural test the experimenter would ask the subjects for permission to proceed. If the subject agreed the examiner would move to the next step. If the subject refused the behavioural test was terminated. Subjects remained seated throughout the test. The eight steps were as follows: (1) The experimenter brings the snake in the aquarium into the room 13 feet from the subject; (2) The snake is moved to a point 8 feet from the subject; (3) The snake is moved to a point 4 feet from the subject; (4) The snake is moved to a point 1 foot from the subject; (5) The aquarium is placed on the subject's lap; (6) The snake is removed from the aquarium and held by the experimenter 1 foot from the subject; (7) The subject touches the snake for $45 \mathrm{~s}$ while the experimenter holds it; (8) The subject holds the snake for $45 \mathrm{~s}$.

Exposure treatments occurred in two 50 -min sessions which were held 2 to 7 days apart. All subjects were given the same treatment rationale which explained that fear is a learned response and that the best way to unlearn this response is by repeatedly facing up to the thing that one fears. Each treatment session started with brief relaxation instructions after which a baseline measure of heart rate was taken. There were a total of ten exposure trials, four in session one and six in session two. Each exposure trial lasted $3 \mathrm{~min}$. In the imaginal 
exposure condition this included 2 mins during which the scene was described and $1 \mathrm{~min}$ of imagery. For the in vivo exposure condition this involved $3 \mathrm{~min}$ of exposure to the snake. Subjects were given $2 \mathrm{~min}$ to relax between each exposure trial.

In the imaginal exposure condition, subjects were presented with two scenes to imagine. Each scene involved a frightening encounter with a snake and included descriptors of behavioural, somatovisceral, and cognitive fear responses. Scene one was presented in the first six exposure trials (the four exposure trials of session one and the first two trials of session two). Scene two was presented during the last four trials of session two.

In vivo subjects were presented with the snake in the same aquarium used in the behavioural test at a distance of approximately 3 feet for the first six trials (the four exposure trials of session one and first two trials of session two). For the last four trials of session two the aquarium was placed on the subject's lap. For each in vivo exposure trial the experimenter left the subject alone with the snake. The experimenter removed the snake from the room between exposure trials.

Scene one and presentation of the snake at the farther distance will be referred to as exposure one. Scene two and presentation of the snake at the closer distance will be referred to as exposure two. Subjects remained seated in a comfortable chair throughout the sessions. All instructions, including imaginal exposure scripts, were presented to subjects via pre-recorded audiotapes. Measures of activation, within session habituation, and across session habituation were based on the following process variables.

Activation was assessed by comparing heart rate during the first trials of exposure one and exposure two to heart rate at baseline assessment for sessions one and two respectively. If heart rate was significantly greater during trial one, compared to baseline level, it was taken to indicate that activation had occurred. Fear ratings were also examined for indices of fear activation.

Within-session habituation was measured by comparing heart rate and fear ratings during trial one to those during trial four for each exposure. A significant decrease indicated habituation. Trend analyses were also utilized to evaluate within session habituation. A significant linear trend for trials one through four would suggest habituation across trials (within session habituation). Across-session habituation was measured in two ways. Trial five of exposure one was actually the first exposure trial for the second treatment session. Across-session habituation can be inferred if there is less activation of fear responses for this trial compared with trial one. Heart rate activation (i.e., the difference between baseline heart rate and heart rate during the exposure trial) for trial five was compared to heart rate activation for trial one. Significantly less activation for trial five would suggest across-session habituation of the heart rate response. Across-session habituation was also measured by compar- 
$i_{B}$ fear ratings for trial one and trial five. If trial five fear ratings were Si gnificantly lower than those for trial one, it would indicate across session habituation.

Subjects' fear of snakes was reassessed after treatment using the same Theasures as during pre-treatment assessment (i.e., SPQ, SNFR, BTAV, B TFR, BTHR).

Results

Pre-treatment equivalence conditions

Inaginal and in vivo conditions were compared based on their scores on the five Measures of snake fear at pretreatment assessment. No significant differences emerged. Subjects in the two conditions, therefore, did not differ in level of snake fear prior to treatment.

Process measures

Predicted differences on the process measures between exposure trials and between an exposure trial and baseline assessment were examined using one-tailed $t$-tests. Since no group differences were predicted on the process measures, two tailed $t$-tests were utilized in making comparisons between exposure groups.

\section{Activation}

For exposure one, heart rate during trial one was significantly higher than during baseline assessment for both the imaginal $(t(16)=3.25, P<0.005$ ) and in vivo $(t(18)=3.39, P<0.005)$ exposure conditions. Heart rate for each trial of exposure one is presented in Fig. 1. Although mean heart rate during trial one appears greater for the imaginal exposure than for the in vivo condition, the difference is not statistically significant. Mean heart rates for trial one suggest that activation occurred in both the imaginal and the in vivo conditions. The fear ratings for trial one of exposure one also suggest activation (see Fig. 2). The mean subjective fear ratings for the imaginal and in vivo exposure conditions were 5.06 and 3.16 respectively. Imaginal exposure subjects rated their level of fear significantly higher than in vivo subjects ( $t(34)$ $=3.06, P<0.005$, two-tailed).

For exposure two, heart rate during trial one was significantly higher than baseline heart rate level for the imaginal exposure condition only $(t(16)=$ $2.66, P<0.01)$. The in vivo subjects did not show a significant increase in heart rate over baseline level. Mean heart rates for all four trials of exposure two are presented in Fig. 3. Examination of fear ratings for each trial of exposure two also suggest that there was activation of fear responses for the imaginal 


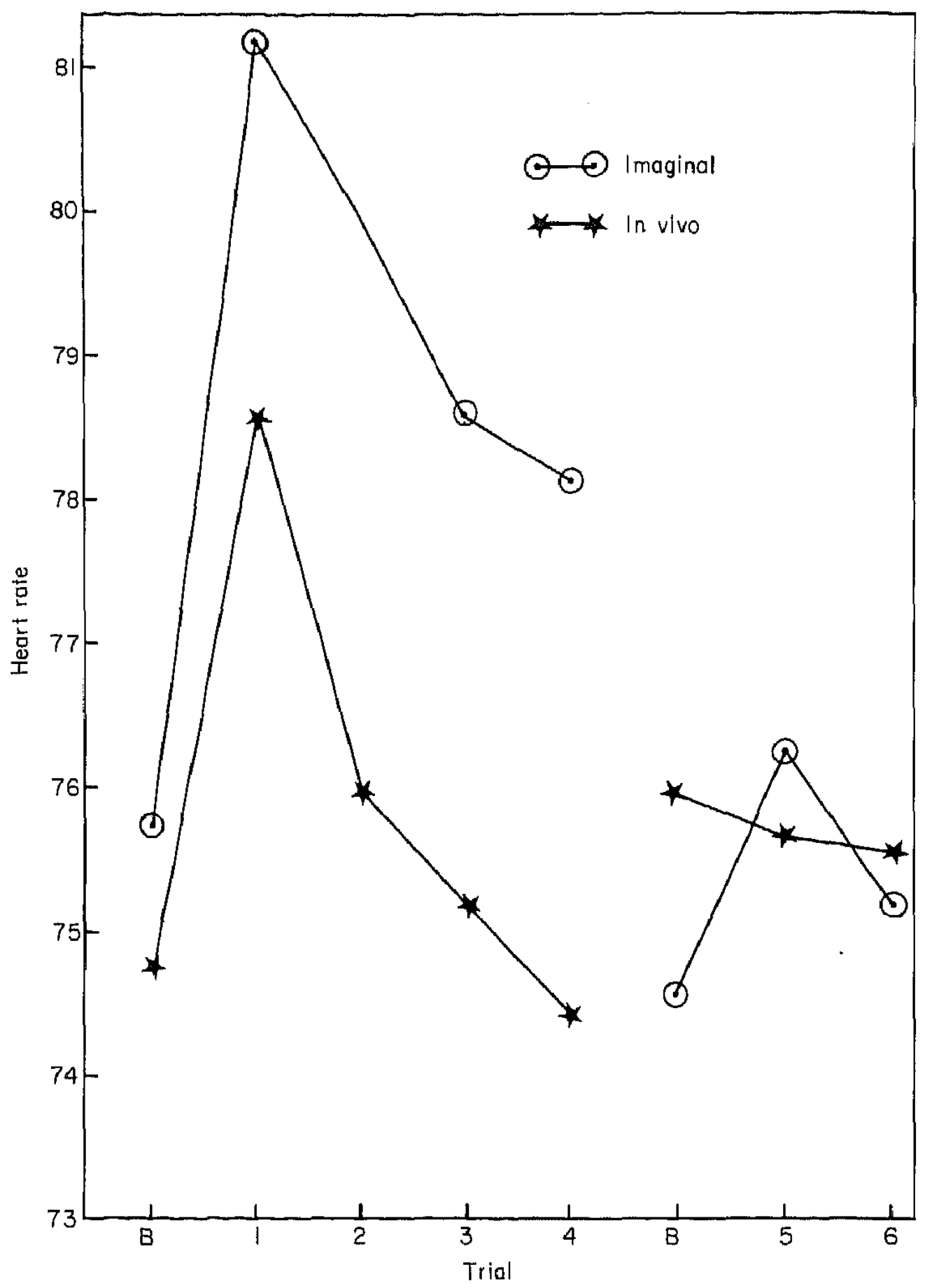

FIGURE 1. Heart rate for exposure one: Baselines and trials 1 through 6 .

exposure but not the in vivo exposure subjects (see Fig. 4). The mean fear rating for the imaginal exposure condition was 4.88 whereas it was only $2 \cdot 21$ for the in vivo condition. This difference in group means is significant $(t(34)=6.08$, $P<0.001$, two-tailed test).

Witbin-session babituation

The heart rate data for exposure one suggest that there was habituation across 


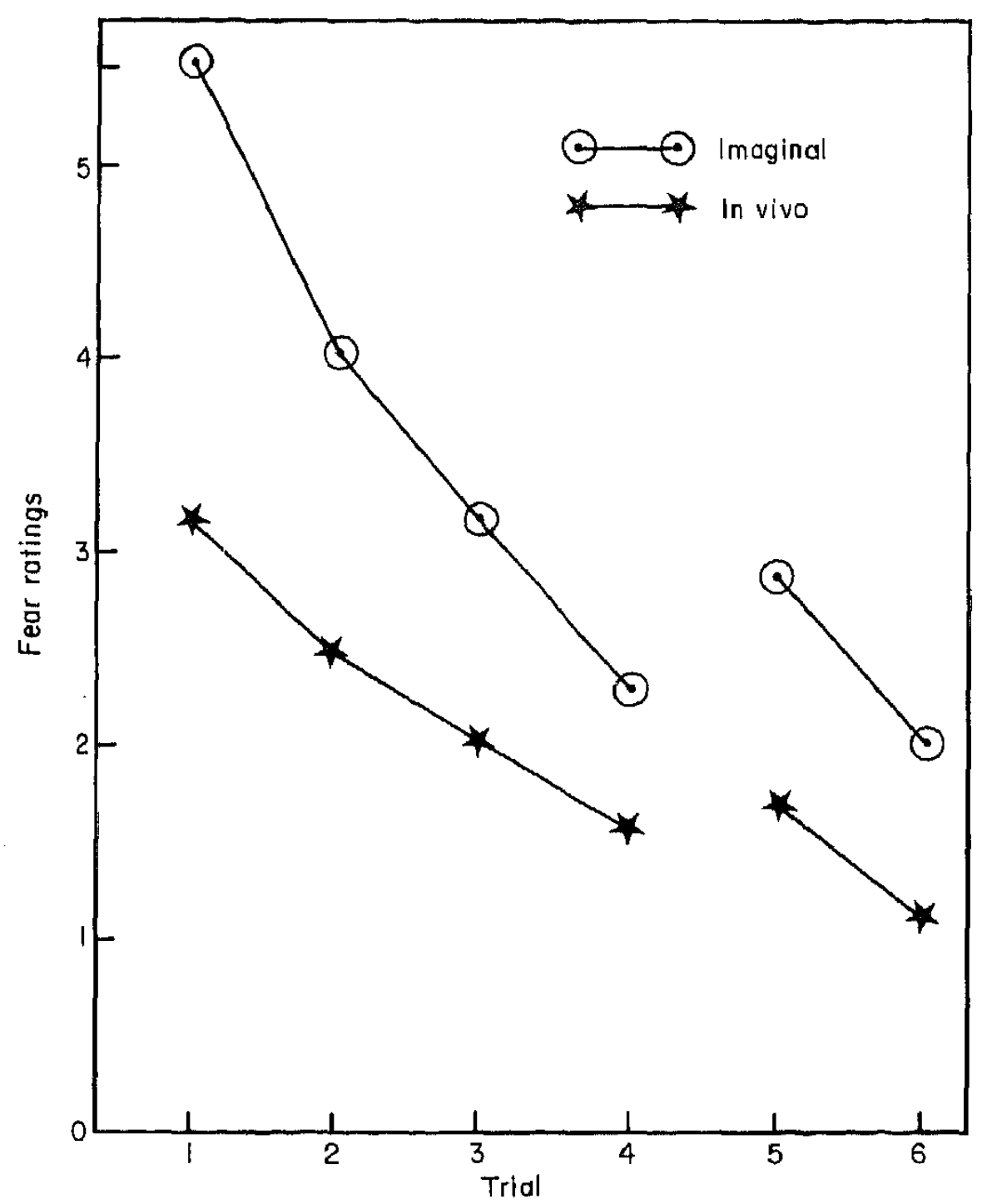

FIGURE 2. Fear ratings for exposure one: Trials 1 through 6.

exposure trials of the physiological fear response for the in vivo exposure condition. Mean heart rate for trial one was significantly greater than for trial four $(t(18)=2.88, P<0.005)$ in the in vivo condition. Within session habituation for this condition is further suggested by a significant linear trend in the data $(F(1,16)=7 \cdot 9, P<0.02)$. For the imaginal exposure condition, heart rate data do not suggest complete within-session habituation. The difference between heart rate for trial one and trial four was non-significant for the imaginal exposure subjects. Furthermore, mean heart rate at trial four was still significantly greater than baseline levels $(t(16)=1.75, P<0.05)$. While there appears to be a linear trend in the data (see Fig. 1), the trend did not reach traditionally accepted levels of statistical significance $(F(1,16)=$ $3 \cdot 26, P<0 \cdot 10$ ). It seems, therefore, that within-session habituation of heart rate activation was incomplete for the imaginal exposure group. 


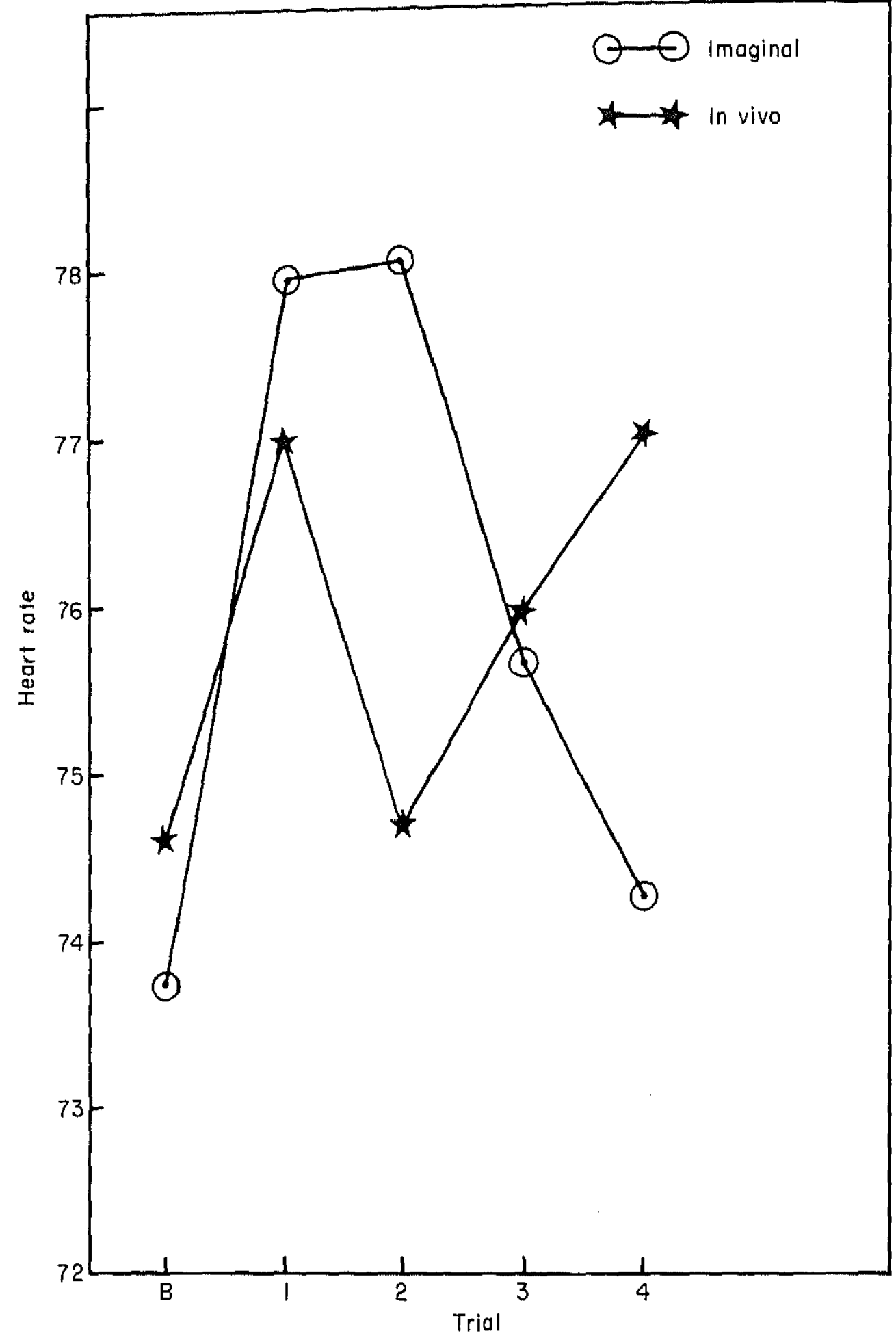

FIGURE 3. Heart rate for exposure two: Trials 1 through 4.

When fear ratings are used as the measure of activation and habituation, within session habituation is seen in both conditions. Mean fear ratings for trial one are greater than those for trial four for both the imaginal $(t(16)=7 \cdot 3$, $P<0.001)$ and in vivo $(t(18)=4.32, P<0.001)$ conditions. Trend analyses 


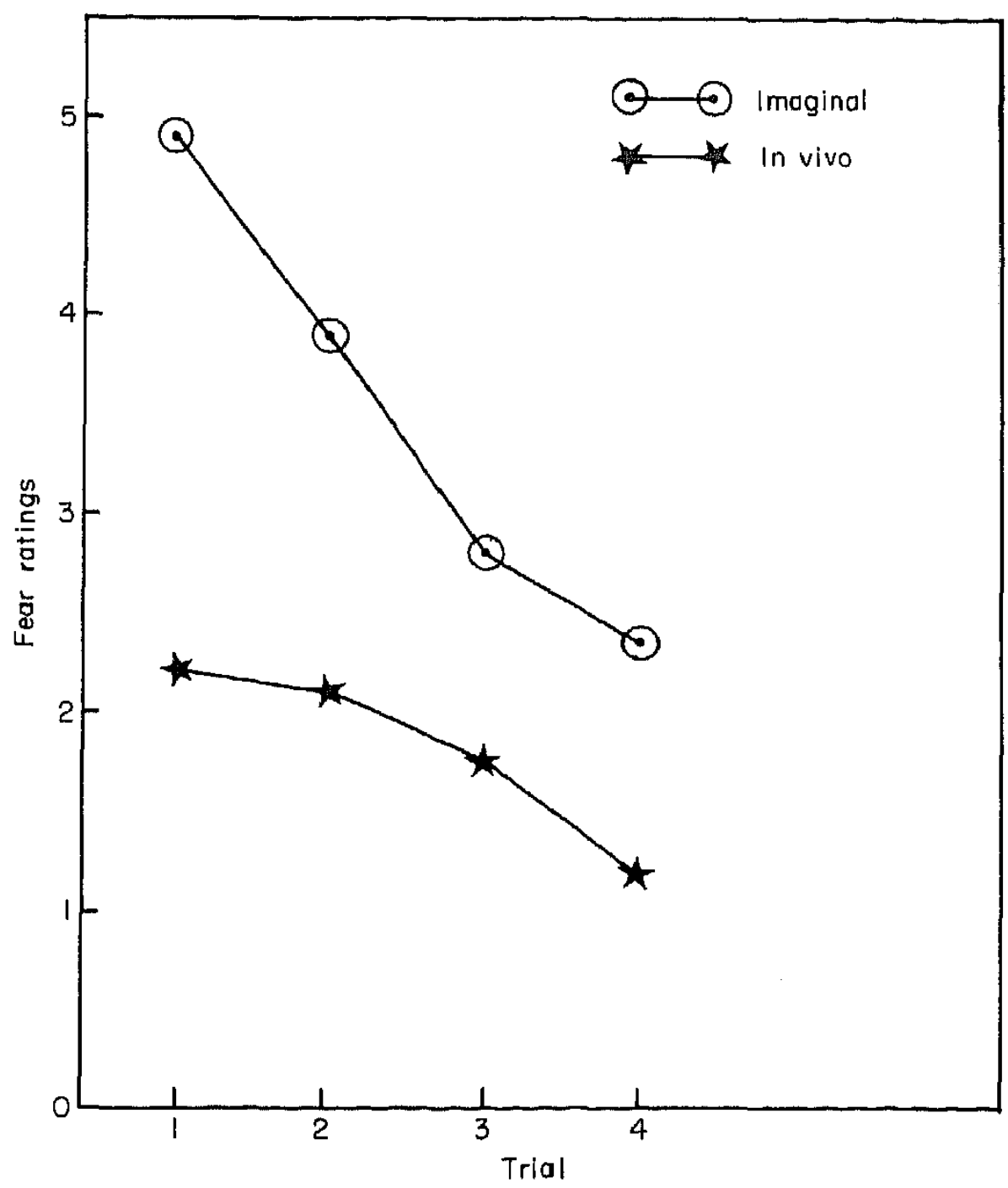

FIGURE 4. Fear ratings for exposure two: Trials 1 through 4.

indicate significant linear trends in fear ratings across trials for both conditions (imaginal: $(F(1,16)=38.1, P<0.001$; in vivo: $F(1,17)=17.7$, $P<0.002)$.

For exposure two, within-session habituation was found for the heart rate data for the imaginal exposure subjects. Mean heart rate at trial one was significantly greater than at trial four $(t(16)=2.67, P<0.01$ ) and a significant linear trend across the four trials was found $(F(1,16)=7.9$, $P<0.02$ ). Neither effect was found for the in vivo condition (see Fig. 3).

Fear ratings suggest within session habituation for both conditions. Imaginal exposure subjects' fear ratings for trial one are significantly greater than for trial four $(t(16)=5.89, P<0.01)$. Trends analysis indicated a significant linear trend across trials $(F(1,16)=38.1, P<0.001)$. Similarly, in vivo subjects rated themselves as significantly less fearful during trial four when 
compared with trial one $(t(17)=4.01, P<0.002)$. A significant linear trend across trials is also apparent in their mean fear ratings $(F(1,17)=17 \cdot 7$, $P<0.002)$. Mean fear ratings for exposure two are presented in Fig. 4.

\section{Across-session babituation}

Heart rate activation for trial five was significantly less than for trial one in both conditions (imaginal: $t(16)=2.33, P<0.02$; in vivo: $t(18)=1.88$, $P<0.04)$. Across-session habituation of heart rate activation, therefore, appears to have occurred in subjects treated with imaginal and in vivo exposure.

Mean fear ratings across trials suggest that across session habituation of subjective fear ratings occurred for both conditions. Mean fear ratings for trial five are significantly lower than for trial one (imaginal: $t(16)=5 \cdot 37$, $P<0.001$; in vivo: $t(18)=3.56, P<0.002)$. Furthermore, the difference between fear ratings for trials four and five are nonsignificant for both conditions.

\section{Outcome}

The five measures of snake fear were analysed using a doubly multivariate repeated measures analysis of variance (SPSS, 1983). A two (exposure condition: imaginal versus in vivo) by two, (trials: pre versus post) design was utilized. The multivariate exposure condition by trials interaction was nonsignificant. Univariate analyses of variance, therefore, need to be interpreted with caution. A significant exposure condition by trials interaction was found for the SPQ $(F(1,34)=6.51, P<0.02)$. This interaction was nonsignificant for all the other measures of snake fear. Examination of pre- and post-treatment means on the SPQ for both treatment conditions indicates that the in vivo condition showed greater improvement on this measure (see Table 1).

The multivariate analysis of variance yielded a significant trials effect (Wilks Lambda $(5,30)=24.74, P<0.001$ ) which indicates that when both conditions are taken together there is significant change in the dependent measures from pre- to post-treatment. Significant univariate trials effects were

TABLE 1. Snake phobia questionnaire: group means and standard deviations assessment

\begin{tabular}{lcc}
\hline Exposure condition & Pre & Post \\
\hline Imaginal & $16 \cdot 88$ & $14 \cdot 06$ \\
& $(5 \cdot 02)$ & $(5 \cdot 27)$ \\
In vivo & $15 \cdot 10$ & $9 \cdot 05$ \\
& $(2 \cdot 75)$ & $(4 \cdot 62)$ \\
\hline
\end{tabular}


found for the SPQ $(F(1,34)=54.74 P<0.001)$; SNFR $(F(1,34)=24 \cdot 74$, $P<0.001)$; BTFR $(F(1,34)=127.93, P<0.001)$; and BTAV $(F(1,34)=$ $76.35, P<0.001$ ) but not for the heart rate measure (BTHR). The significant univariate trials effect for the SPQ cannot, of course, be interpreted directly since there is a significant exposure condition by trials interaction. A series of $t$-tests for the other measures of snake fear were conducted for the two treatment conditions separately. The results indicate that both conditions led to improvement on these measures of snake fear.

\section{Discussion}

In this study, indices of emotional processing including activation, withinsession habituation, and across-session habituation were revealed during imaginal and in vivo exposure treatment of simple phobics. Both treatments engendered similar patterns of activation and habituation of fear responses, with some interesting differences. First, subjects tended to show greater activation of fear responses during imaginal exposure, although differences in activation did not always reach statistically significant levels. These differences in activation can be understood from the emotional processing perspective. Imaginal exposure scenes involved catastrophic encounters with snakes. The descriptions of the snakes in these scenes clearly indicated that they were dangerous. This description would presumably contain information that matched the meaning propositions of the subjects' fear network. Meaning propositions are the phobic individual's interpretations of the other components of the fear structure (e.g., "snakes are dangerous"). Since the imaginal exposure scenes contained propositons which matched those in the fear network, activation of fear responses should occur (Lang, 1984). In contrast, in vivo subjects encountered a relatively docile snake that was securely caged. While this presentation would provide ample stimulus information matching the fear network, few meaning propositions matching subjects' propositional representations of snakes would be available and hence activation would be less likely. An alternative explanation for the relatively weaker activation of fear responses occasioned by in vivo exposure might lie in the specific methods of this study. The same snake was used in the in vivo exposure session as had been presented during the behavioural test. Therefore, the first treatment session was actually the second exposure session for these subjects. Hence, some activation and habituation may already have occurred.

The second difference between the imaginal and in vivo exposure conditions on the indices of processing is that there was activation of fear responses during exposure two for imaginal but not in vivo subjects. This difference was likely due to the fact that the second imaginal flooding scene was markedly different 
than scene one, whereas the second presentation of the snake was only slightly different than the first (i.e., the same snake as in exposure one was presented during exposure two only at a closer distance). In the in vivo condition, habituation to these particular snake stimuli had already occurred by exposure two.

Finally, the imaginal and in vivo exposure conditions differed in the speed with which within-session habituation occurred. In vivo exposure was associated with quicker habituation of fear arousal within treatment sessions. In fact, within-session habituation was not complete for imaginal exposure subjects in the first treatment session. The linear trend in the data suggest that withinsession habituation would have occurred had additional exposure trials been utilized. The failure to allow for complete within-session habituation may be related to the group difference observed on one of the outcome measures.

There were, in fact, few between group differences observed in treatment outcome. On only one of the four measures of snake fear which showed a significant decrease across trials did a condition by trial interaction emerge. In vivo exposure subjects improved more on the questionnaire measure of snake fear, but not on the behavioural and self-report measures gathered during the behavioural test, or on the global rating. This lack of strong group differences is not surprising given that emotional processing occurred in both treatments. This is consistent with the findings of our review of the imaginal versus in vivo exposure literature (Hecker and Thorpe, 1987). Our examination of six studies comparing imaginal and in vivo exposure in the treatment of clinically significant fear indicated that successful treatment was related to activation and habituation of fear responses, regardless of the specific treatment technique employed. Although the present study utilized an analogue population, our earlier review of the literature revealed the same pattern of activation and habituation when clinically anxious groups were studied. Procedural differences may be important only to the extent that certain procedures facilitate emotional processing more than others. As Richards (1988) has pointed out, if the patterns of anxiety responses during imaginal and in vivo exposure are the same in simple phobias, "clinically there should be nothing to choose between the two procedures" (p. 209).

One problem in applying emotional processing as an explanatory construct is making the distinction between process and outcome assessment. It could be argued that activation, within-session habituation, and across-session habituation are simply descriptors of treatment outcome at various points during exposure therapy. Foa and Kozak (1986) suggest that emotional processing might be distinguished from treatment outcome by the generality of the concepts: "emotional processing of fear is indicated only by measures of that fear, treatment outcome is a broader concept. It also includes functioning 
indirectly related to the fear structure that is hypothesized to change as a result of fear reduction, such as, job performance, social interaction, sleep disturbance, and general mood state" (p. 22). A limitation of the present study is the paucity of information collected with respect to treatment outcome as defined in this broad manner. What data there are point toward in vivo exposure as having a stronger effect upon outcome. The SPQ was the most general assessment instrument used, since, among other things, it asks subjects whether or not they would engage in behaviours that would place them at risk for encountering snake (e.g., "I avoid going to parks or on camping trips because there may be snakes about"). In vivo exposure subjects improved more on this measure.

One challenge for future investigators in this area will be to develop alternative methods of assessing the hypothesized fear structure that are relatively distinct from treatment outcome. Clinical researchers have looked to cognitive psychology for help in developing methodologies to assess the fear network. The dichotic listening procedure (Foa and McNally, 1986) and Stroop color-naming task (Hope, Rapee, Heimberg and Dumbeck, in press) have been utilized recently to assess various aspects of anxiety states. While this work is in its early stages of development it promises to provide fruitful information regarding the fear reduction process.

\section{Acknowledgement}

Critical reviews of earlier drafts of this manuscript were contributed by Geoffrey L. Thorpe. His criticisms and suggestions, as well as those of the anonymous reviewers and the Editor, are gratefully acknowledged.

\section{References}

FOA, E. G. and KozAK, M. J. (1986). Emotional processing of fear: Exposure to corrective information. Psychological Bulletin 99, 20-35.

FOA, E. G. and MCNALlY, R. J. (1986). Sensitivity to feared stimuli in obsessivecompulsives: A dicholtic listening analysis. Cognitive Therapy and Researcb 10, $477-485$.

Hecker, J. E., Port, E. and Thorpe, G. L. (1986, November). Imagery Training, Imagery Content and Type of Exposure: An Investigation of Lang's Bioinformational Theory, Paper presented at the 20th Annual Meeting of the Association for the Advancement of Behaviour Therapy. Chicago. IL.

HeCker, J. E. and Thorpe, G. L. (1987). Fear reduction processes in imaginal and in vivo flooding: A comment on James' review. Behavioural Psychotherapy 15, $215-223$.

Hope, D. A., Rapee, R. M. Heimberg, R. G. and Dombeck, M. J. Representations of the self in social phobia: Vulnerability to social threat. Cognitive Therapy and Research (in press). 
Klorman, R., Weerts, T. C., Hastings, J. E., Melamed, B. G. and Lang, P. J. (1974). Psychometric description of some specific fear questionnaires. Bebavior Therapy 5, 401-419.

LANG, P. J. (1979). A bio-informational theory of emotional imagery. Psychophysiology $16,495-51$.

LANG, P. J. (1984). Cognition in emotion: Concept and action. In C. Izard, J. Kagan and R. Zajonc (Eds), Emotion, Cognition and Behavior. New York: Cambridge University Press. (pp. 192-225).

LANG, P. J. (1985). The cognitive psychophysiology of emotion: Fear and anxiety. In A. H. Tuma and J. D. Maser (Eds.), Anxiety and the Anxiety Disorders. Hillsdale, N.J.: Lawrence Erlbaum. (pp. 131-170).

RichARDS, D. A. (1988). The treatment of snake phobia by imaginal exposure. Behavioural Psychotberapy 16, 207-216.

SPSS, Inc. (1983). SPSSx User's Guide. New York: McGraw-Hill.

(Date received: 30 May 1989) 